\section{LA-UR-11-03399}

Approved for public release; distribution is unlimited.

\title{
Grid Expansion Planning for Carbon Emissions Reduction
}

Author(s):
Intended for:

Russell Bent G. Loren Toole

\section{Power Engineering Society General Meeting 2012}

\section{- LosAlamos \\ NATIONAL LABORATORY}

EST. 1943

Los Alamos National Laboratory, an affirmative action/equal opportunity employer, is operated by the Los Alamos National Security, LLC for the National Nuclear Security Administration of the U.S. Department of Energy under contract DE-AC52-06NA25396. By acceptance of this article, the publisher recognizes that the U.S. Government retains a nonexclusive, royalty-free license to publish or reproduce the published form of this contribution, or to allow others to do so, for U.S. Government purposes. Los Alamos National Laboratory requests that the publisher identify this article as work performed under the auspices of the U.S. Department of Energy. Los Alamos National Laboratory strongly supports academic freedom and a researcher's right to publish; as an institution, however, the Laboratory does not endorse the viewpoint of a publication or guarantee its technical correctness.

Form $836(7 / 06)$ 


\title{
Grid Expansion Planning for Carbon Emissions Reduction
}

\author{
Russell Bent, and G. Loren Toole
}

\begin{abstract}
In recent years the grid expansion planning problem has become increasingly complex and challenging. The integration of renewable generation is a source of many of these challenges. These challenges often include a deficiency in transmission capacity in regions with high potential for renewable energy production. Historically, this lack of capacity has had adverse effects such as negative price market conditions or the curtailing of other green generation sources. This paper considers a combined generation and transmission expansion model to avoid the curtailment of existing green generation sources, in other words maximize the realized carbon reduction of adding renewable generation. Recent work on Randomized Constructive Heuristics (RCH) has shown this approach to be quite effective in addressing the Transmission Network Expansion Planning (TNEP) problem. In this paper, we propose a generalization of RCH to handle simultaneous carbon reduction and expansion cost objectives as well as multi-scenario planning.
\end{abstract}

Index Terms-Carbon Emissions, Generation Expansion Planning, Transmission Network Expansion Planning, Simulation Optimization, Non-linear Optimization, Local Search.

\section{INTRODUCTION}

In recent years that has been increasing interest in integrating generation from renewable sources, such as wind and solar, into existing electric power grids [1], [2]. Much of this interest has risen from a desire to reduce the carbon footprint of power grids. However, increasing the penetration of renewable generation has presented a number of challenges, for example, fluctuating power production and a deficiency in transmission capacity in desirable wind and solar locations. In the latter case, a lack in transmission capacity (along with other factors) can lead to undesirable outcomes, such as negative power production prices [3] and the curtailment of other carbon neutral generators. In short, it is possible for grid operations to reduce the benefits of expansion.

This paper presents a grid expansion planning problem for upgrading an existing power grid to integrate specified renewable generation while at the same time minimizing the carbon output of the grid and minimizing construction costs. In other words, we seek to maximize the carbon impact of adding renewable generation capacity. This problem is variant of the well studied Transmission Network Expansion Planning (TNEP) [4], [5], [6], [7], Generation Expansion Planning (GEP) [8], [9], [10], [11], and Integrated Resource Planning (IRP) [12], [13], [14] problems.

In this paper we adopt the Simulation-Optimization algorithm of [13], [15] to address this problem. The key idea of the approach is the encapsulation of the power model within a simulation black box. The algorithm is allowed

The authors are with the Decision Applications and Computational \& Statistical Sciences Divisions of Los Alamos National Laboratory (LANL). to query the black box for power flow information about proposed expansion plans. The algorithm generalizes existing constructive heuristics [16], [17] within a randomized search tree exploration procedure. It is referred to as a Randomized Constructive Heuristic ( $\mathrm{RCH})$. In short, the key contributions of this paper include:

- A combined transmission and generation expansion planning model for minimizing carbon emissions of a power grid.

- A generalization of RCHs to solve the carbon minimization problem.

- A generalization of RCHs to solve multi-scenario grid expansion planning problems

- Case studies demonstrating that expansion planning can be used to achieve near best case carbon reductions when adding renewables

- Case studies showing that adding renewable generation solely based on expansion cost can have unexpectedly low reductions in carbon output

- A demonstration that the simulation-optimization approach of [15] can handle optimal power flow models, as was conjectured

The key point of this paper is to assume renewable generation expansion decisions are pre-determined: the challenge is how to deploy them to maximize carbon reductions.

Literature Review While literature on Power Grid Expansion Planning (PGRP) goes back many decades, it has only been recently, with a few exceptions [18], that there has been interest in how to use planning to reduce carbon emissions [9], [14], [19]. We now briefly discuss some of these contributions in order to place this paper in the context of the existing literature.

More specifically, in reference [9], the authors described a linear programming model for the GEP. It includes carbon emissions in a multi-objective formulation. It also evolves the grid expansion over multiple time periods. It differs from this paper in that transmission systems are represented by a transportation model, transmission expansions are not part of the model, and it does not model multiple operational scenarios.

Reference [8] also includes minimizing carbon emissions as part of a multi-objective formulation. This paper is one of the few that models scenarios of possible operational modes when determining expansion plans (in this case, component failures). They include transmission expansions on an inter-area level, where the transmission system is radial (a transportation model). The remaining transmission system is assumed to have enough capacity. It also assumes a mixed-integer programming (MIP) formulation. Reference [19] also shares some 
similarities with this paper. It includes a combined generation and transmission model that has carbon emissions as part of a multi-objective function. Though they use a linearized DC model, as they use a genetic algorithm it could be extended to more complex power flow models. They consider a single scenario that aggregates the Mexican power grid.

Reference [14] presents a multi-objective formulation that focuses on a joint generation expansion and transmission expansion (between areas in the grid) problem. It evolves the system over time but does not consider operational scenarios. The paper uses a transportation model for transmission flows in order to keep the model tractable for mathematical programming techniques.

Reference [20] also considers carbon reduction as a criteria for grid expansion planning. They propose a generation only expansion model and balance the cost to build different types of generation units and their carbon output (among other criteria). They do not consider how the new and old generation is dispatched in the solutions. Reference [21] proposes a generation expansion planning model that reduces carbon by considering a variety of technologies, such as carbon capture and new renewable generation. The transmission system is assumed to have sufficient capacity and it is not modeled. References [22], [23], [24] provide overviews of methods for modeling expansion planning with multiple objective functions. They provide survey information on how decision makers weight cost with carbon emissions and other objectives for making generation mix decisions (no power system modeling).

In general, the literature has focused on developing linear mathematical programming models for computational tractability. Here, we use a more general algorithm to ensure the ability to use more complex models, when the need arises.

The remainder of this paper is organized as follows. Section II formally defines the PGRP problem. Section III describes the algorithm used to generate expansion plans. Section IV discusses the experimental results and Section V concludes this paper

\section{Problem Definition}

Buses The PGRP problem is described in terms of a set of buses, $\mathcal{B}$, that represent geographically located nodes in a power network e.g. generators, loads, and substations. Each bus, $i$, is defined by parameters $G_{i}, L_{i}, \iota_{i}^{-}, \iota_{i}^{+}$which represent its generation, load (demand for power), minimum voltage (per unit) and maximum voltage (per unit). $P\left(G_{i}^{+}\right), P\left(G_{i}^{-}\right), Q\left(G_{i}^{+}\right)$, and $Q\left(G_{i}^{-}\right)$are used to denote the maximum real, minimum real, maximum reactive, and minimum reactive components of existing generation. Similarly, $P\left(L_{i}\right)$ and $Q\left(L_{i}\right)$ are used to denote the desired real and reactive components of load. The decision variable $c_{i}$ is used to define the renewable generation added to $i . c_{i}$ has discrete domain $\left\{c_{i}^{-}, c_{i}^{-}+\delta, \ldots, c_{i}^{+}-\delta, c_{i}^{+}\right\}$, where $\delta$ is the generation increment. This generation is modeled as must take. It is also assumed enough name plate capacity and/or storage is included in the construction to achieve the specified level of generation. $\mu_{i}$ is used to denote the $\mathrm{CO} 2$ per MWH of existing generation at $i$.
Transmission Corridors The PGRP is also described by a set of edges, $\mathcal{E}$, called transmission corridors, connecting pairs of nodes. A transmission corridor $i, j$ between buses $i$ and $j$ has a decision variable $c_{i, j}$ that defines the number of circuits (power lines) in the corridor. The variable has discrete domain $\left\{c_{i, j}^{-}, c_{i, j}^{-}+1, \ldots, c_{i, j}^{+}-1, c_{i, j}^{+}\right\}$where $c_{i, j}^{-}$is defined as the number of circuits the corridor starts with. $c_{i, j}^{-}=c_{i, j}^{+}$when no circuits may be added to a corridor. A circuit is also defined by parameter $i, j$ which denotes the capacity of a single circuit in the corridor. Similarly, $r_{i, j}, x_{i, j}$, and $\psi_{i, j}$ denote the resistance, reactance, and capacity of a single circuit in the corridor.

PGRP Solution A transmission network solution, $\sigma$, is defined as a set of variable assignments $\bigcup_{i \in \mathcal{B}} c_{i} \leftarrow d_{i} \cup \bigcup_{i, j \in \mathcal{E}} c_{i, j} \leftarrow$ $d_{i, j}{ }^{1}$, where $d_{i}$ is drawn from the domain of $c_{i}$ and $d_{i, j}$ is drawn from the domain of $c_{i, j}$. By convention, unassigned variables are assumed to be $c_{i}^{-}$and $c_{i, j}^{-}$, respectively. $\sigma\left(c_{i}\right)$ and $\sigma\left(c_{i, j}\right)$ are used to denote the variable assignments for $\sigma$. Simulation TNEP algorithms have at their disposal a simulator $\mathcal{S}$ for determining the power flows for $\sigma . \mathcal{S}(\sigma)$ returns true when it is able to compute the flows. $\mathcal{S}_{\digamma_{i, j}}(\sigma)$ denotes the flow in corridor $i, j, \mathcal{S}_{g_{i}}(\sigma)$ denotes the amount of existing generation capacity used at bus $i, l_{i}$ denotes the load met at $i$, and $\mathcal{S}_{v_{i}}(\sigma)$ the voltage at bus $i$. For simplicity, this notation is shortened to $\digamma_{i, j}, g_{i}, l_{i}$, and $v_{i}$ when $\mathcal{S}(\sigma)$ is understood from context.

A TNEP solution $\sigma$ is feasible when the following constraints are satisfied, i.e.

$$
\begin{cases}c_{i, j}^{-} \leq c_{i, j} \leq c_{i, j}^{+} & (i, j \in \mathcal{E}) \\ c_{i}^{-} \leq c_{i} \leq c_{i}^{+} & (i \in \mathcal{B}) \\ \mathcal{S}(\sigma)=\text { true } & \\ \sum_{i \in \mathcal{B}} c_{i}=C & \end{cases}
$$

Constraints (1) and (2) represent the limits on the possible expansions. Constraint (3) enforces that $\mathcal{S}$ is able to solve the model (for example, when AC models are used). Finally, constraint (4) ensures that $C$ MWs of renewable generation output is added to the system.

Physical constraints are relaxed and incorporated into the objective function (similar to Lagrangian Relaxation). ${ }^{2}$ The physical violations of $\sigma$ are calculated as the sum of flow that exceeds the capacity of the circuits, the sum of voltages that fall below $\iota_{i}^{-}$or above $\iota_{i}^{+}$, the sum of generation out of bounds, and the sum of load shed, i.e.

$$
\begin{aligned}
\eta(\sigma)= & \sum_{i, j \in \mathcal{E}} \max \left(0, \digamma_{i, j}-\psi c_{i, j}\right)+ \\
& \sum_{i \in \mathcal{B}} \max \left(0, \iota_{i}^{-}-v_{i},{ }_{i}^{+}-v_{i}\right)+ \\
& \sum_{i \in \mathcal{B}} \max \left(0, g_{i}-g_{i}^{+}, g_{i}^{-}-g_{i}\right)+ \\
& \sum_{i \in \mathcal{B}} \max \left(0, L_{i}-l_{i}\right) .
\end{aligned}
$$

The $\mathrm{CO} 2$ of $\sigma$ is calculated as $\zeta(\sigma)=\sum_{i \in \mathcal{B}} \mu_{i} g_{i}$. Finally, the cost of $\sigma$ is defined by $\kappa(\sigma)=\sum_{i, j \in \mathcal{E}} c_{i, j} \kappa_{i, j}+\sum_{i \in \mathcal{B}} c_{i} \kappa_{i}$ where $\kappa_{i, j}$ is the cost of building a circuit in corridor $i, j$ and $\kappa_{i}$ is the cost of building generation at bus $i$. The objective

\footnotetext{
${ }^{1}$ The notation $c_{i} \leftarrow d_{i}$ is used to denote the assignment of value $d_{i}$ to variable $c_{i}$

${ }^{2}$ In this paper the DC optimal power flow model is used (DCOPF) and these constraints can be directly incorporated into the optimization model. However, in the spirit of the simulation optimization of [13], [15], the constraints are kept in the simulation so that in future work, more complex models of power systems (such as AC) can be used.
} 
function, $f(\sigma)$, is then a lexicographic, multi-objective function of the form $\min f(\sigma)=\langle\eta(\sigma), \zeta(\sigma), \kappa(\sigma)\rangle$.

\section{RCH ALGORITHM}

To solve this problem we adopt the Randomized Constructive Heuristic ( $\mathrm{RCH})$ algorithm of [15] and for completeness it is redescribed here. This algorithm is used due to its ability to accommodate arbitrary complex models of $\mathcal{S}$, its ability to generalize existing PGRP constructive heuristics and the high quality results it has achieved on other models of expansion planning [13], [15]. The algorithm is discussed in Figure 1. The function $\mathrm{RCH}$ describes the randomization of the constructive heuristic $(\mathrm{CH})$. Line 3 orders the possible expansion variables according to the $\mathrm{CH}$ (function ORDERVARIABLE). Lines 4-5 chooses a variable randomly from the ordering, where the randomness is determined by the parameter $\omega$. As $\omega \leftarrow \infty$ the more likely the $\mathrm{CH}$ is followed exactly. Line 6 orders the variable assignments of the variable according to the following function: When $\eta(\sigma)>0$ the domain is ordered by component additions, no change $(\sigma(\mathcal{X}))$, and component removals, i.e. ${ }^{3}$

$$
\left\langle\sigma(\mathcal{X})+1,, \mathcal{X}^{+}, \sigma(\mathcal{X}), \sigma(\mathcal{X})-1,, \mathcal{X}^{-}\right\rangle
$$

otherwise it is ordered in reverse, i.e.

$$
\left\langle\sigma(\mathcal{X})-1, \mathcal{X}^{-}, \sigma(\mathcal{X}), \sigma(\mathcal{X})+1,, \mathcal{X}^{+}\right\rangle
$$

Lines 6-7 chooses a variable assignment randomly from the ordering where the randomness is determined by parameter $\gamma$. As $\gamma \leftarrow \infty$ the more likely the variable order is followed exactly. Line 8 updates the solution according to the variable assignment. Parameters $\alpha$ and $\beta$ are used as stopping criteria for the heuristic when variable assignments do not lead to improving solutions. $\alpha$ determines how many times in a row the solution may degrade and $\beta$ determines how many times in a row $\mathcal{S}$ may fail to obtain a solution. $\mathcal{S}$ is implicitly executed in line 8 during the variable assignment. Lines 1 and 2 determine if the stopping criteria is met. Lines 9-14 update the stopping criteria. Lines 15 returns the best solution discovered during the course of the search.

$\mathrm{RCH}$ is executed $P$ times in the function OPTIMIZEPGRP (lines 4-8). The RCH procedure is initialized with the best solution discovered and repeated until an improving solution is no longer found (line 9). In this paper, the function ORDERVARIABLES is implemented using the constructive heuristic of [16], [17], [25], which has worked well in practice within the $\mathrm{RCH}$ implementation of [15]. More formally the function is defined by:

$\left\langle\mathcal{X}_{1}, \mathcal{X}_{2}, \ldots, \mathcal{X}_{n}\right\rangle: f\left(\sigma \cup\left[\sigma\left(\mathcal{X}_{i}\right) \leftarrow d_{1}\left(\mathcal{X}_{i}\right)\right]\right) \leq f\left(\sigma \cup\left[\sigma\left(\mathcal{X}_{i+1}\right) \leftarrow d_{1}\left(\mathcal{X}_{i+1}\right)\right]\right.$

where $d_{1}(\mathcal{X})$ is used as shorthand to denote the first element of $\operatorname{ORDERDOMAIN}(\mathcal{X})$. This function orders the variables by the variable assignment that will improve the objective function the most.

\footnotetext{
${ }^{3} \sigma(\mathcal{X})$ is used to denote the assignment of a value to variable $y$ in $\sigma$. Thus, $\sigma(\mathcal{X})+1$ is the next largest variable assignment of $\sigma$.
}

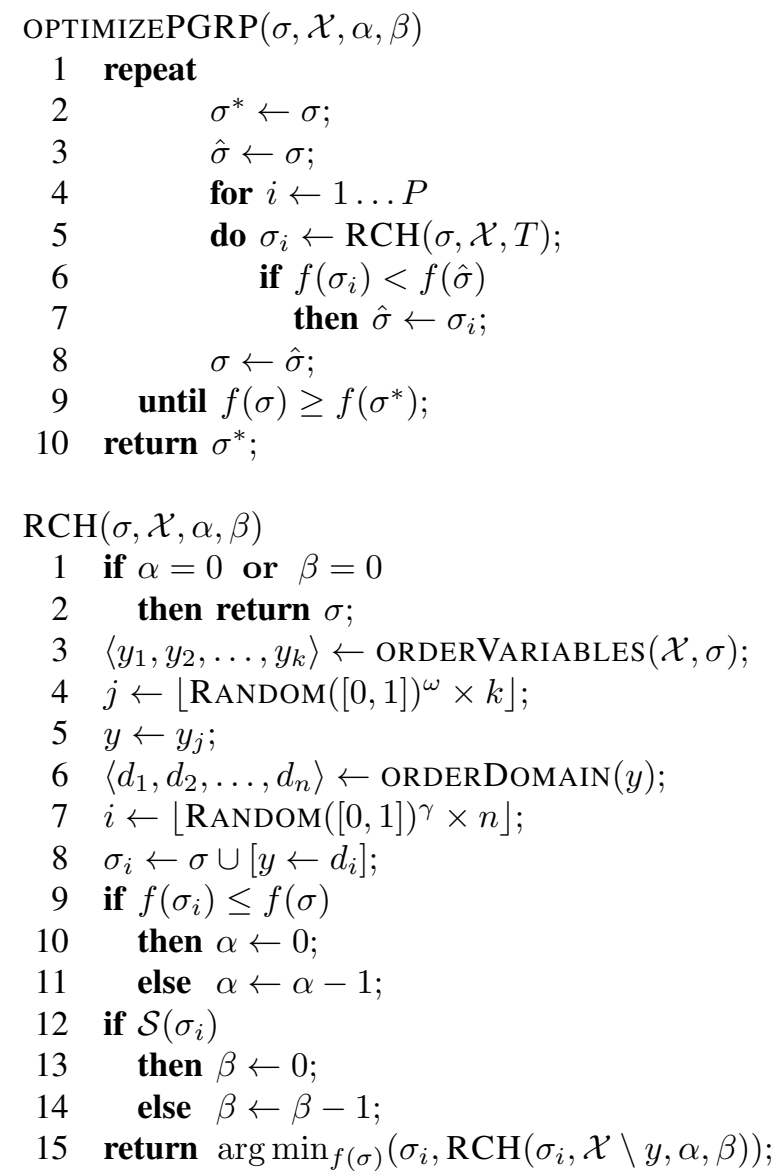

Fig. 1. Randomized constructive heuristic (RCH)

\section{EXPERIMENTAL RESUltS}

In order to evaluate our approach we consider three different case studies. The first case study adapts the 24 bus IEEE RTS-79 problem [26] to expand transmission and renewable generation to replace carbon output in the model. The second case study adapts the transmission expansion problems of [27] to include renewable generation. The third case study considers a multi-scenario variation of the second case study. In all three cases the linearized DC optimal power flow (OPF) model is used for the implementation of $\mathcal{S}$. This allows us to determine if expansion can target carbon reductions under an economic model for grid operations (generation usage).

\section{A. RTS-79 Benchmark}

The first case study adapts the RTS-79 problems of [26]. Up to three additional circuits are allowed in each corridor. Three circuits may also be added to the new corridors suggested in [27]. The costs of adding circuits are found in [27]. The fuel types for each generator are also contained in [28]. Based on these fuel types, costs are calculated based on reference [29] and carbon output per MWH are based on reference [30]. These numbers are reported in Table I. In the case of multiple generators at a bus, without loss of generality we average cost and carbon output, weighted by capacity. 
TABLE I

GENERATOR OPERATIONS COST (\$ PER MWH) AND CARBON EMISSIONS (LBS PER MWH)

\begin{tabular}{c|c|c||c|c|c} 
Bus & Cost & Carbon & Bus & Cost & Carbon \\
\hline 1 & 142 & .00247 & 16 & 101.0 & .00201 \\
2 & 142 & .00247 & 18 & 110.0 & 0 \\
7 & 300 & .00170 & 21 & 110.0 & 0 \\
13 & 300 & .00163 & 22 & 58.5 & 0 \\
15 & 156 & .00202 & 23 & 101.0 & .00220
\end{tabular}

TABLE II

RESULTS ON PROBLEM RTS-79

\begin{tabular}{c|c|c||c|c|c}
$C$ & $\zeta$ & $\kappa$ & $C$ & $\zeta$ & $\kappa$ \\
\hline 100 & 3.54 & $0 \mathrm{~K}$ & 500 & 2.82 & $0 \mathrm{~K}$ \\
200 & 3.37 & $0 \mathrm{~K}$ & 1000 & 1.77 & $0 \mathrm{~K}$ \\
300 & 3.21 & $0 \mathrm{~K}$ & 2000 & 0.84 & $66 \mathrm{~K}$ \\
400 & 2.99 & $0 \mathrm{~K}$ & 2700 & 0.00 & $81 \mathrm{~K}$
\end{tabular}

Eight versions of the problem for $C=$ $100,200,300,400,500,1000,2000$, and 2700 (constraint 4) were considered. The renewable generation can be added to each of buses $4,6,11,17$, and 20 (totaling up to $C$ ) in increments of $100 \mathrm{MW}$. Based on the results of [15], $\alpha=\beta=3$, and $\gamma=\omega=10$. As $C$ is fixed and it is assumed the capacity factors are homogeneous, without loss of generality we assume the cost of adding generation is 0 . It also assumed enough nameplate capacity and/or storage is installed to achieve the desired output.

Table II provides the results on the RTS-79 model. As can be seen here, the network is able to accommodate up to $1000 \mathrm{MW}$ of new renewable generation (that displaces carbon emitters) without expansion. At that point, a handful of circuits need to be added to the system. At $2700 \mathrm{MW}$, the grid does not produce any carbon, which is not surprising, as the load is $2700 \mathrm{MW}$ and the renewable generation is "must take."

Table III provides the solutions to the problem. The first column provides the value of parameter $C$. The next 5 columns provide the amount of renewable generation added at each bus. The last column provides the circuits that were added.

\section{B. TNEP Benchmarks}

The second case study uses the transmission expansion planning problems of [27], which are based on the RTS-79 problem. There are 4 problems, G1, G2, G3, and G4 and the details are found in [27]. In each problem, up to three new circuits are allowed in each existing corridor. There are

TABLE III

SOLUTIONS TO THE RTS-79 PROBLEMS. COLUMNS 2-6 INDICATE THE AMOUNT OF GENERATION ADDED IN MW AND THE LAST COLUMN THE CIRCUITS ADDED.

\begin{tabular}{c|c|c|c|c|c|c}
$C$ & 4 & 6 & 11 & 17 & 20 & Circuit \\
\hline 100 & 100 & - & - & - & - & - \\
200 & 100 & - & 100 & - & - & - \\
300 & 100 & - & 100 & 100 & - & - \\
400 & 400 & - & - & - & - & - \\
500 & 300 & 100 & - & 100 & - & - \\
1000 & 100 & 100 & 600 & 100 & 100 & - \\
2000 & 200 & 300 & 100 & 100 & 1300 & $(2,8)$ \\
& & & & & & $(2,8)$ \\
2700 & 300 & 100 & 1900 & 200 & 100 & $(3,9)$ \\
& & & & & & $(9,11)$
\end{tabular}

TABLE IV

Carbon and Construction Cost Results on problem G1, G2, G3, G4.

\begin{tabular}{c|c|c|c|c|c|c|c|c|} 
& \multicolumn{2}{|c|}{$\mathrm{G} 1$} & \multicolumn{2}{c|}{$\mathrm{G} 2$} & \multicolumn{2}{c|}{$\mathrm{G} 3$} & \multicolumn{2}{c}{$\mathrm{G} 4$} \\
$C$ & $\zeta$ & $\kappa$ & $\zeta$ & $\kappa$ & $\zeta$ & $\kappa$ & $\zeta$ & $\kappa$ \\
\hline 100 & 9.96 & $878 \mathrm{~K}$ & 10.27 & $718 \mathrm{~K}$ & 12.10 & $459 \mathrm{~K}$ & 11.43 & $906 \mathrm{~K}$ \\
200 & 9.71 & $482 \mathrm{~K}$ & 10.06 & $927 \mathrm{~K}$ & 11.86 & $368 \mathrm{~K}$ & 11.19 & $462 \mathrm{~K}$ \\
300 & 9.52 & $478 \mathrm{~K}$ & 9.88 & $904 \mathrm{~K}$ & 11.63 & $234 \mathrm{~K}$ & 10.98 & $383 \mathrm{~K}$ \\
400 & 9.32 & $578 \mathrm{~K}$ & 9.55 & $614 \mathrm{~K}$ & 11.37 & $266 \mathrm{~K}$ & 10.78 & $483 \mathrm{~K}$ \\
500 & 9.11 & $797 \mathrm{~K}$ & 9.46 & $551 \mathrm{~K}$ & 11.14 & $250 \mathrm{~K}$ & 10.57 & $586 \mathrm{~K}$ \\
1000 & 8.18 & $508 \mathrm{~K}$ & 8.10 & $496 \mathrm{~K}$ & 9.90 & $395 \mathrm{~K}$ & 9.69 & $410 \mathrm{~K}$ \\
2000 & 6.89 & $410 \mathrm{~K}$ & 7.56 & $334 \mathrm{~K}$ & 8.18 & $325 \mathrm{~K}$ & 8.52 & $248 \mathrm{~K}$ \\
3000 & 4.95 & $526 \mathrm{~K}$ & 6.77 & $385 \mathrm{~K}$ & 7.38 & $250 \mathrm{~K}$ & 7.09 & $280 \mathrm{~K}$
\end{tabular}

also seven new corridors where up to three circuits may be added. The generation costs and carbon output are the same as Table I. In this problem, up to $3000 \mathrm{MW}$ of renewable generation in $100 \mathrm{MW}$ increments can be added to each of buses $1,2,7,13,15,16,18,21,22$, and 23 (existing generation sites). Eight versions of each problem are considered for $C=100,200,300,400,500,1000,2000$, and 3000 (constraint 4). This problem is also interesting because even before expansion is applied, the models contain physical violations. Thus, these must be addressed at the same time as cost and carbon output and we can compare with solutions that do not include $\zeta$ in the objective. Once again, based on the results of [15], $\alpha=\beta=3$ and $\gamma=\omega=10$. As the amount of added generation, $C$, is fixed, we assume the cost of adding generation is 0 .

Table IV shows the results obtained by $\mathrm{RCH}$ on these problems. All 4 problems show a steady decrease in the amount of carbon that is produced $(\zeta)$. The expansion costs tend to fluctuate when carbon emissions. With this objective, the plan that is selected is the one that reduces carbon the most (curtails the heavy carbon emitters the most when the OPF is executed). As such, cost has limited impact on the plan that is selected and will not necessarily follow any particular trend.

Figures 2 and 3 drill down into the results of G1 in order to understand how carbon output impacts the solution quality. Figure 2 compares a number of different approaches by consider $\zeta$ (y axis) as $C$ increases. The minimize carbon line shows the results of $\mathrm{RCH}$ including $\zeta$ in $f(\sigma)$. The minimize cost line shows the results of $\mathrm{RCH}$ when $\zeta$ is not included in $f(\sigma)$. The lower bound and upper bound lines show the bounds for $\zeta$ (the heaviest and lightest carbon emitters curtailed respectively).

There are a number of important observations to make here. First, $\mathrm{RCH}$ (minimize carbon) is able to achieve results very near the lower bound, indicating that transmission expansion combined with appropriate placement of renewable generation can achieve nearly the best case impact. Second, as seen by the minimize cost results, when $\kappa$ is the driver of expansion, adding renewable generation has less of an impact on reducing $\zeta$. Third, even without $\zeta$ the costs still fluctuate to certain degree, mainly as a side-effect of the "must take" generation. At some levels of $C$, the additional generation can help alleviate congestion and at some levels it contributes to congestion, not unlike what was seen in [3].

The ability to reduce $\zeta$ does come at a cost, as seen in Figure 3. Here it can be seen that the objective of reducing 


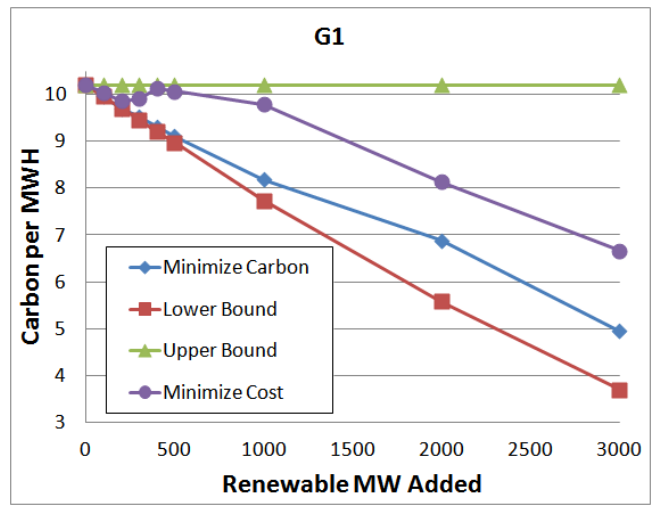

Fig. 2. A comparison of $\zeta$ when carbon output is an objective and when it is not.

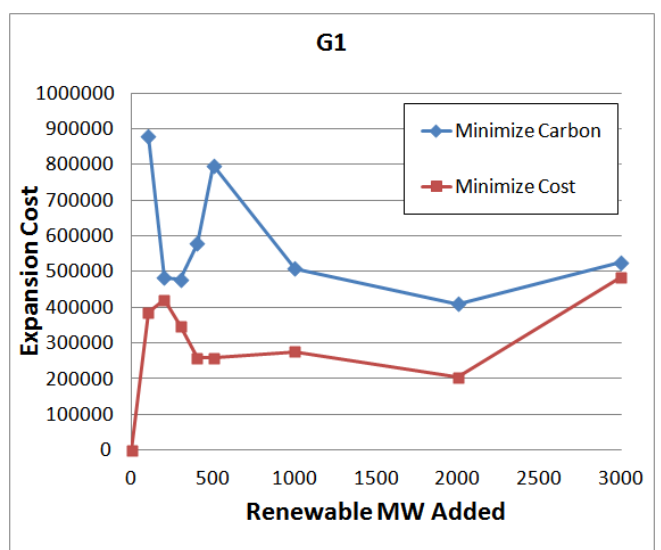

Fig. 3. A comparison of $\kappa$ when carbon out is an objective and when it is not.

$\zeta$ can make the cost of expansion as much as four times more expensive. However, without accepting this cost, adding renewable generation may not have the carbon emissions impact that is expected (Figure 2). The solutions are provided in the appendix. Interestingly, generation is rarely added where existing carbon free generation is located (buses 18, 21, and 22 ), indicating that the search does not desire to curtail such generation with a lack of capacity. It also does not simply incrementally add renewable generation to the high carbon emitters (otherwise buses 1 and 2 would have $1000 \mathrm{MW}$ of new generation).

\section{Multi Scenario Benchmark}

The third case study also uses the transmission expansion planning problems of [27]. This time the four problems are treated together as a single multi-scenario problem. They vary in the amount of existing generation and load. The objective is to find a solution that minimizes $f$ on average across all 4 scenarios. Eight versions of the problem are considered for $C=100,200,300,400,500,1000,2000$, and 3000 (constraint 4). Based on the results of [15], $\alpha=\beta=3$ and $\gamma=\omega=$ 10. Once again, as $C$ is fixed, we assume the cost of adding generation is 0 .

The results for the multi-scenario problem are shown in
TABLE $\mathrm{V}$

RESULTS ON THE MULTI-SCENARIO BENCHMARK. $\zeta$ IS THE AVERAGE POUNDS OF CARBON PER MW ACROSS ALL FOUR SCENARIOS. $\kappa$ IS THE CONSTRUCTION COSTS.

\begin{tabular}{c|c|c||c|c|c}
$C$ & $\zeta$ & $\kappa$ & $C$ & $\zeta$ & $\kappa$ \\
\hline 100 & 10.9775 & $630 \mathrm{~K}$ & 500 & 10.140 & $876 \mathrm{~K}$ \\
200 & 10.750 & $899 \mathrm{~K}$ & 1000 & 9.128 & $655 \mathrm{~K}$ \\
300 & 10.640 & $844 \mathrm{~K}$ & 2000 & 7.425 & $676 \mathrm{~K}$ \\
400 & 10.335 & $1131 \mathrm{~K}$ & 3000 & 6.280 & $472 \mathrm{~K}$
\end{tabular}

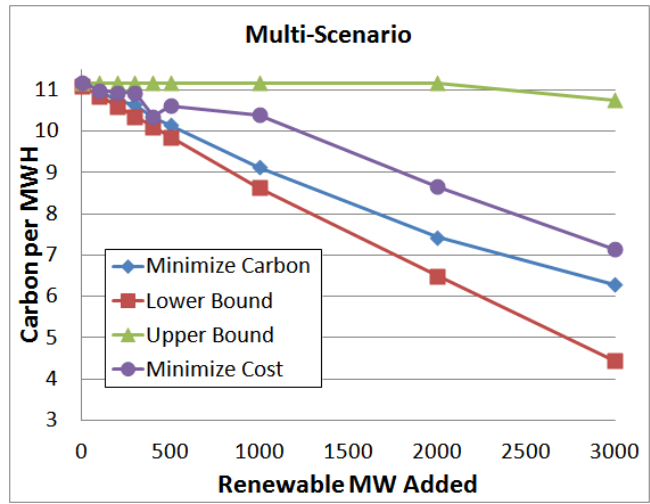

Fig. 4. Comparison of different approaches on $\zeta$ as $C$ increases.

Table XII in the appendix. In this case $\zeta$ is the average carbon output for the four scenarios. Once again, a steady carbon reduction is achieved as $C$ is increased. Interestingly, there is a more consistent cost reduction as $C$ increases than there was in the individual scenarios (the average carbon is higher than each scenario individually). This indicates the scenario specific solutions do not provide good solutions to the other scenarios for $\zeta$, and a solution across all scenarios is more appropriate. Indeed, it shows that when there is a diversity of scenarios, the maximum carbon reductions for each scenario cannot be achieved without impacting the other scenarios.

Figures 4 and 5 compare the solutions for when $\zeta$ is included in $f(\sigma)$ and when it is not. In short, in the multiscenario problem, the cost sacrifice to minimize $\zeta$ is not extreme as was seen in Figure 3. This provides evidence that under multi-scenario situations, maximum carbon reduction can be achieved without overly large additional costs. The

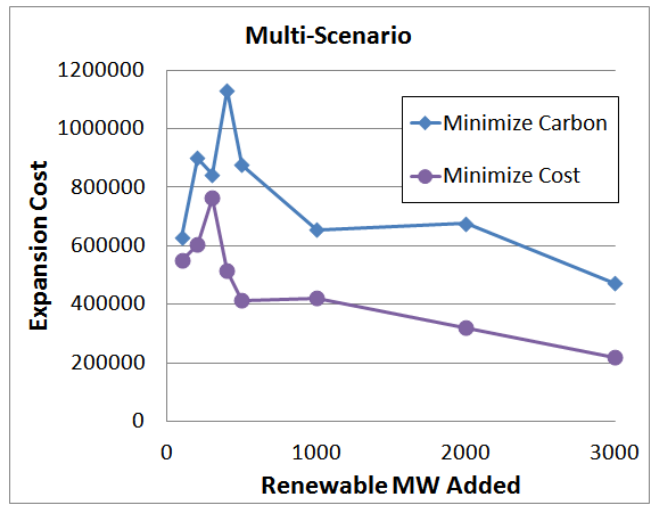

Fig. 5. Comparison of different approaches on $\zeta$ as $C$ increases 
need to generate a solution that is good for multiple scenarios smooths out the expansion costs (albeit at a higher level).

\section{CONClusion}

Recent trends and future projections indicate a widespread adoption of green generation, such as wind and solar, is occurring. Generally speaking, the expectation is that adopting these technologies will decrease the carbon footprint of existing power grids. However, as this paper has shown, it is important to ensure that renewable generation is added carefully in conjunction with transmission in order to ensure that renewable generation actually displaces carbon emitting generators when renewable energy is available. Without such considerations, the addition of renewable generation can have a minimal impact on the grids carbon output as it can displace existing green generation, making the benefit negligible.

There remains a number of interesting questions to address in future work, including case studies that consider real power grids that are considering the addition of renewable generation. In particular, the multi-scenario case study discussed in this paper suggest future work to include power output from renewable generation in order to expand grids so that they exploit spatial diversity in generation output from renewable sites (generation expansion costs should be included in the model). It will also be interesting to include operation's cost in the expansion objective, is this could further exasperate the carbon emissions discrepancy. Finally, it will be interesting to consider how large-scale storage might play a role in this expansion scenario, in particular, ensuring that the addition of storage actually helps reduce the carbon output to its maximum benefit.

\section{ACKNOWLEDGMENTS}

This work was supported by the LANL LDRD project Optimization and Control Theory for Smart Grids.

\section{REFERENCES}

[1] J. Lawhorn, D. Osborn, J. Caspary, B. Nickel, D. Larson, W. Lasher, and M. E. Rahman, "The view from the top," IEEE Power and Energy Magazine, vol. November/December, pp. 76-88, 2009.

[2] J. Achenbach, "The 21st century grid: Can we fix the infrastructure that powers our lives?" National Geographic Magazine, pp. 118-140, July 2010.

[3] R. Baldick, "Wind energy and electricty markets," University of Texas, Tech. Rep. National Academy of Engineering Regional Meeting, 2009.

[4] B. Hobbs, "Optimization methods for electric utility resource planning," European Journal of Operational Research, vol. 83, pp. 1-20, 1995.

[5] J. McCalley, R. Kumar, O. Volij, V. Ajjarapu, H. Liu, L. Jin, and W. Zhang, "Models for transmission expansion planning based on reconfigurable capacitor switching," in Electric Power Networks, Efficiency, and Security. John Wiley and Sons, 2006.

[6] K. Nara, "State of the arts of the modern heuristics application to power systems," IEEE Power Engineering Society Winter Meeting, 2000, vol. 2, pp. 1279-1283, 2000 .

[7] G. Latorre, R. D. Cruz, J. M. Areiza, and A. Villegas, "Classification of publications and models on transmission expansion planning," IEEE Transactions on Power Systems, vol. 18, no. 2, pp. 938-946, 2003.

[8] H. Tekiner, D. Coit, and F. Felder, "Multi-period multi-objective electricity generation expansion planning problem with monte carlo simulation," Electric Power Systems Research, vol. 80, no. 12, pp. 1394-1405, 2010.

[9] J. C. Meza, M. Yildirim, and A. Masud, "A model for the multiperiod multiobjective power generation expansion problem," IEEE Transactions on Power Systems, vol. 22, pp. 871-878, 2007.
[10] J. Sirikum, A. Techanitissawad, and V. Kachitvichyanukul, "A new efficient ga-bender's decomposision method: For power generation expansion planning with emission controls," IEEE Transactions on Power Systems, vol. 22, no. 3, pp. 1092-1100, 2007.

[11] A. Kagiannas, D. Askounis, and J. Psarras, "Power generation planning: A survey from monopoly to competition," Electrical Power and Energy Systems, vol. 26, pp. 413-421, 2004.

[12] J. H. Roh, M. Shahidehpour, and Y. Fu, "Market-based coordination of transmission and generation capacity planning," IEEE Transactions on Power Systems, vol. 22, no. 4, pp. 1406-1419, 2007.

[13] R. Bent, A. Berscheid, and G. L. Toole, "Generation and transmission expansion planning for renewable energy integration," in Power Systems Computation Conference (PSCC), Stockholm, Sweden, 2011.

[14] C. Unsihuay-Vila, J. Marangon-Lima, A. Z. d. Souza, and I. PerezArriaga, "Multistage expansion planning of generation and interconnections with sustainable energy development criteria: A multiobjective model," Electrical Power and Energy Systems, vol. 33, pp. 258-270, 2011.

[15] R. Bent and W. B. Daniel, "Randomized discrepancy bounded local search for transmission expansion planning," in IEEE Power Engineering Society Meeting (PES), Detroit, Michigan, 2011.

[16] E. Bustamante-Cedeno and S. Arora, "Multi-step simultaneous changes constructive heuristic algorithm for transmission network expansion planning," Electric Power Systems Research, vol. 79, pp. 586-594, 2009.

[17] R. Romero, C. Rocha, J. Mantovani, and I. Sanchez, "Constructive heuristic algorithm for the DC model in network transmission expansion planning," in IEEE Proceedings of Generation, Transmission and Distribution, vol. 152, 2005, pp. 277-282.

[18] Y.-C. Kim and B.-H. Ahn, "Multicriteria generation-expansion planning wih global environmental considerations," IEEE Transactions on Engineering Managment, vol. 40, no. 2, pp. 154-161, 1993.

[19] J. L. C. Meza, M. B. Yildirim, and A. S. M. Masud, "A multiobjective evolutionary programming algorithm and its applications to power generation expansion planning," IEEE Transactions on Systems, Man, and Cybernetics - Part A Systems and Humans, vol. 39, no. 5, pp. 10861096, 2009.

[20] C. H. Antunes, A. G. Martins, and I. S. Brito, "A multiple objective mixed integer linear programming model for power generation expansion planning," Energy, vol. 29, pp. 613-627, 2004.

[21] Q. Chen, C. Kang, Q. Xia, and J. Zhong, "Power generation expansion planning model towards low-carbon economy and its application in china," IEEE Transactions on Power Systems, vol. 25, no. 2, pp. 1117$1124,2010$.

[22] P. Linares and C. R. , "A multiple criteria decision making approach for electricity planning in spain: Economic versus environmental objectives," Journal of Operational Research Society, vol. 51, pp. 736-743, 2000.

[23] G. Mavrotas and D. Diakoulaki, "A mixed integer multiple objective linear programming model for capacity expansion in an autonomous power generation system," in Energy and Environment, 2005, pp. 191210.

[24] N. Voropai and E. Y. Ivanova, "Multi-criteria decision analysis techniques in electric power system expansion planning," Electrical Power and Energy Systems, vol. 24, pp. 71-78, 2002.

[25] M. Rider, A. Garcia, and R. , "Power system transmission network expansion planning using AC model," IET Generation, Transmission and Distribution, vol. 1, no. 5, pp. 731 - 742, 2007.

[26] Reliability Test System Task Force of the Application of Probability Methods Subcommittee, "IEEE reliability test system," IEEE Transactions on Power Apparatus and Systems, vol. PAS-98, no. 6, pp. 20472054, 1979.

[27] R. Feng and D. Hill, "A new strategy for transmission expansion in competitive electricity markets," IEEE Transactions on Power Systems, vol. 18, no. 1, pp. 374-380, 2003.

[28] Reliability Test System Task Force, "The IEEE reliability test system 1996," IEEE Transactions on Power Systems, vol. 14, no. 3, pp. 1010$1020,1996$.

[29] United States Energy Information Administration, "Annual energy outlook," Department of Energy, Tech. Rep., 2011.

[30] United States Energy Information Admistration, "Carbon dioxide emissions from the generation of electric power in the united states," Department of Energy, Tech. Rep., 2000.

Russell Bent Russell Bent received his $\mathrm{PhD}$ in Computer Science from Brown University in 2005 and is currently a staff scientist at LANL in the 
Energy and Infrastructure Analysis Group. His publications include deterministic optimization, optimization under uncertainty, infrastructure modeling and simulation, constraint programming, algorithms, and simulation. Russell has published 1 book and over 30 articles in peer-reviewed journals and conferences in artificial intelligence and operations research. A full list of his publications can be found at http://public.lanl.gov/rbent/.

G. Loren Toole G. Loren Toole serves as the task lead for several infrastructure projects at LANL. He is responsible for network interdependency and asset prioritization studies delivered to Federal customers including DOE, DHS and DOD. His engineering design, planning and construction experience extends over 30 years, with specialized experience in electric utility planning; renewables project development and design and applying state-of-the-art tools used by electric utilities for generation, transmission and distribution analysis. He earned degrees in Electrical Engineering (BS E.E. and MS E.E.) from the Georgia Institute of Technology and serves as an Adjunct Professor, University of Missouri College of Engineering (Columbia)

\section{APPENDIX}

TABLE VI

Solutions to Problems G1,G2,G3,G4 (Minimize Carbon) Circuit ADDITIONS

\begin{tabular}{|c|c|c|c|c|c|c|c|c|}
\hline C & 100 & 200 & 300 & 400 & 500 & 1000 & 2000 & 3000 \\
\hline$(1,2)$ & $0,0,0,0$ & $0,0,0,0$ & $0,0,0,3$ & $0,0,0,3$ & $0,0,0,0$ & $0,0,3,0$ & $0,0,1,0$ & $\overline{0,0,0,0}$ \\
\hline$(1,3)$ & $0,0,0,0$ & $0,0,0,0$ & $0,0,0,0$ & $0,0,0,0$ & $0,0,0,0$ & $1,0,0,0$ & $0,0,0,0$ & $0,0,0,0$ \\
\hline$(1,5)$ & $0,1,0,0$ & $0,0,0,0$ & $0,0,0,0$ & $0,0,0,0$ & $0,1,0,0$ & $0,0,0,0$ & $0,0,0,1$ & $3,0,0,0$ \\
\hline$(1,8)$ & $0,0,0,0$ & $0,0,0,0$ & $0,1,0,0$ & $0,0,0,0$ & $0,0,0,0$ & $0,0,0,0$ & $0,0,0,0$ & $0,0,0,0$ \\
\hline$(2,4)$ & $0,0,0,1$ & $0,0,0,0$ & $0,0,0,0$ & $0,0,0,0$ & $1,0,0,0$ & $0,0,0,0$ & $0,0,0,0$ & $0,0,0,0$ \\
\hline$(2,6)$ & $1,1,0,0$ & $1,1,0,1$ & $1,1,0,0$ & $1,0,0,0$ & $1,1,0,1$ & $0,1,0,1$ & $1,0,0,0$ & $1,1,0,0$ \\
\hline$(2,8)$ & $0,0,0,0$ & $0,1,0,0$ & $0,0,0,0$ & $0,0,0,0$ & $0,0,0,0$ & $0,0,0,0$ & $0,0,0,0$ & $0,0,0,0$ \\
\hline$(3,9)$ & $0,1,2,0$ & $0,0,0,0$ & $0,1,0,0$ & $0,0,0,0$ & $0,1,0,0$ & $0,0,0,0$ & $0,0,0,0$ & $0,0,0,0$ \\
\hline$(3,24)$ & $1,1,0,2$ & $1,1,2,1$ & $1,2,1,1$ & $3,2,1,3$ & $1,1,1,3$ & $0,2,2,1$ & $1,1,1,1$ & $2,1,0,1$ \\
\hline$(4,9)$ & $0,0,0,0$ & $0,0,0,1$ & $0,0,0,0$ & $0,0,0,0$ & $0,0,0,0$ & $0,0,0,0$ & $0,0,0,0$ & $0,0,0,0$ \\
\hline$(5,10)$ & $0,0,1,1$ & $0,0,0,0$ & $0,0,0,0$ & $0,0,0,0$ & $0,0,0,0$ & $1,0,0,0$ & $0,0,0,0$ & $0,0,0,0$ \\
\hline$(6,7)$ & $0,0,0,1$ & $0,2,0,0$ & $0,1,0,0$ & $0,3,0,0$ & $0,1,0,1$ & $1,0,0,1$ & $0,1,0,0$ & $0,1,0,1$ \\
\hline$(6,10)$ & $1,0,1,3$ & $1,0,3,0$ & $0,0,1,2$ & $0,0,2,2$ & $1,0,1,0$ & $0,0,1,0$ & $0,1,1,1$ & $0,0,1,0$ \\
\hline$(7,8)$ & $3,1,2,3$ & $3,1,3,3$ & $3,2,2,3$ & $3,1,3,3$ & $3,1,3,3$ & $3,3,3,3$ & $3,1,3,3$ & $3,1,3,3$ \\
\hline$(8,9)$ & $0,0,0,2$ & $0,0,0,1$ & $0,0,0,0$ & $0,0,0,0$ & $0,0,0,0$ & $0,0,2,0$ & $0,0,0,0$ & $0,1,0,0$ \\
\hline$(9,11)$ & $1,0,0,0$ & $0,0,0,0$ & $0,0,0,0$ & $0,0,0,0$ & $1,0,0,0$ & $0,0,0,0$ & $0,0,0,0$ & $0,0,0,0$ \\
\hline$(9,12)$ & $0,0,0,0$ & $0,0,0,1$ & $0,0,0,0$ & $0,0,0,0$ & $0,0,0,0$ & $0,0,0,1$ & $0,0,0,1$ & $0,1,0,0$ \\
\hline$(10,11)$ & $1,0,1,0$ & $0,0,0,0$ & $0,0,0,0$ & $0,0,0,0$ & $0,0,0,0$ & $0,0,0,0$ & $0,1,0,0$ & $0,0,0,0$ \\
\hline$(10,12)$ & $0,0,0,1$ & $0,1,1,0$ & $0,0,1,1$ & $0,1,1,1$ & $1,0,1,0$ & $1,0,1,0$ & $0,1,1,0$ & $0,0,2,0$ \\
\hline$(11,13)$ & $0,0,1,0$ & $0,0,0,0$ & $0,0,0,0$ & $0,0,0,0$ & $0,0,0,0$ & $0,0,0,0$ & $0,1,0,0$ & $0,0,0,1$ \\
\hline$(11,14)$ & $0,0,0,1$ & $0,0,0,0$ & $0,0,0,0$ & $0,0,0,0$ & $0,0,0,0$ & $0,0,0,0$ & $0,0,0,0$ & $0,0,0,0$ \\
\hline$(12,13)$ & $1,0,0,0$ & $0,0,0,0$ & $0,0,0,0$ & $0,0,0,0$ & $0,0,0,0$ & $0,0,0,0$ & $0,0,0,0$ & $0,0,0,1$ \\
\hline$(12,23)$ & $1,0,0,0$ & $0,0,0,0$ & $0,0,0,0$ & $0,0,0,0$ & $0,0,0,0$ & $0,0,0,0$ & $0,0,0,0$ & $0,0,0,0$ \\
\hline$(13,14)$ & $0,1,0,2$ & $0,0,0,0$ & $0,0,0,0$ & $0,0,0,0$ & $1,2,0,1$ & $0,0,0,0$ & $0,0,0,1$ & $0,0,0,0$ \\
\hline$(14,16)$ & $2,0,1,0$ & $1,0,0,0$ & $1,1,0,0$ & $1,1,0,0$ & $0,0,0,0$ & $1,1,0,1$ & $1,0,0,0$ & $1,1,0,0$ \\
\hline$(14,23)$ & $0,1,0,1$ & $0,1,1,1$ & $0,1,1,1$ & $0,0,1,1$ & $0,0,1,1$ & $0,0,1,0$ & $0,0,1,0$ & $0,0,1,0$ \\
\hline$(15,16)$ & $0,1,0,0$ & $0,0,0,0$ & $0,0,0,0$ & $0,0,0,0$ & $0,0,0,0$ & $0,0,0,0$ & $0,0,0,0$ & $0,0,0,0$ \\
\hline$(15,21)$ & $0,1,0,0$ & $1,3,0,0$ & $2,1,0,0$ & $2,2,0,0$ & $3,1,0,1$ & $1,2,0,0$ & $1,0,0,0$ & $1,0,0,0$ \\
\hline$(15,24)$ & $0,1,0,2$ & $1,1,0,1$ & $1,3,0,1$ & $1,1,0,1$ & $1,1,0,1$ & $0,1,0,1$ & $1,0,1,0$ & $1,1,0,0$ \\
\hline$(16,17)$ & $3,1,1,1$ & $2,1,1,1$ & $1,1,0,1$ & $1,1,0,1$ & $1,1,0,0$ & $3,1,0,1$ & $1,1,0,0$ & $1,0,0,0$ \\
\hline$(16,19)$ & $1,1,0,0$ & $1,1,0,0$ & $1,0,0,0$ & $1,0,0,0$ & $1,1,0,0$ & $1,0,0,0$ & $1,0,0,0$ & $1,0,0,0$ \\
\hline$(16,23)$ & $0,0,0,0$ & $0,1,0,0$ & $0,0,0,0$ & $0,0,0,0$ & $0,0,0,0$ & $0,0,0,0$ & $0,0,0,0$ & $0,0,0,0$ \\
\hline$(17,18)$ & $1,1,0,1$ & $1,0,0,0$ & $0,0,0,0$ & $0,0,0,0$ & $0,0,0,0$ & $1,0,0,0$ & $0,0,0,0$ & $0,0,0,0$ \\
\hline$(17,22)$ & $1,0,0,0$ & $0,0,0,0$ & $0,1,0,0$ & $0,0,0,0$ & $0,0,0,0$ & $0,0,0,0$ & $0,0,0,0$ & $0,0,0,0$ \\
\hline$(18,21)$ & $0,0,1,0$ & $0,0,0,0$ & $0,0,0,0$ & $0,0,0,0$ & $0,0,0,0$ & $0,0,0,0$ & $0,0,0,0$ & $0,0,0,0$ \\
\hline$(19,20)$ & $0,1,0,0$ & $0,0,0,0$ & $0,0,0,0$ & $0,0,0,0$ & $0,0,0,0$ & $0,0,0,0$ & $0,0,0,0$ & $0,0,0,0$ \\
\hline$(19,23)$ & $0,0,1,0$ & $0,1,0,0$ & $0,0,0,0$ & $0,0,0,0$ & $0,0,0,0$ & $0,0,0,0$ & $0,0,0,0$ & $0,0,0,0$ \\
\hline$(21,22)$ & $0,1,0,0$ & $0,0,0,0$ & $0,0,0,0$ & $0,0,0,0$ & $1,0,0,0$ & $0,0,0,0$ & $0,0,0,0$ & $0,0,0,0$ \\
\hline
\end{tabular}

TABLE VII

Solutions to G1 Problem (Minimize Carbon) Generation ADDITIONS IN MW

\begin{tabular}{c|c|c|c|c|c|c|c|c|}
$\mathrm{C}$ & 100 & 200 & 300 & 400 & 500 & 1000 & 2000 & 3000 \\
\hline 1 & - & 100 & 100 & 100 & 100 & 200 & 100 & 800 \\
2 & 100 & - & 100 & 100 & 100 & 100 & 200 & 100 \\
7 & - & 100 & - & 100 & 100 & 400 & 100 & 100 \\
13 & - & - & - & 100 & 100 & - & 100 & 1900 \\
15 & - & - & - & - & 100 & 100 & 100 & - \\
16 & - & - & 100 & - & - & - & 1400 & 100 \\
18 & - & - & - & - & - & 100 & - & - \\
21 & - & - & - & - & - & 100 & - & - \\
22 & - & - & - & - & - & - & - & - \\
23 & - & - & - & - & - & - & - & -
\end{tabular}

TABLE VIII

Solutions to G2 Problem (Minimize Carbon) Generation ADDITIONS IN MW

\begin{tabular}{c|c|c|c|c|c|c|c|c|}
$\mathrm{C}$ & 100 & 200 & 300 & 400 & 500 & 1000 & 2000 & 3000 \\
\hline 1 & - & 100 & - & 100 & 300 & 100 & 200 & 200 \\
2 & 100 & - & - & 100 & - & 1000 & 100 & 600 \\
7 & - & - & 100 & 100 & - & 100 & 100 & 100 \\
13 & - & - & 100 & - & 100 & - & 1400 & 500 \\
15 & - & 100 & 100 & - & 100 & - & 100 & 1300 \\
16 & - & - & - & 100 & - & - & 100 & 100 \\
18 & - & - & - & - & - & - & - & - \\
21 & - & - & - & - & - & - & - & 100 \\
22 & - & - & - & - & - & - & - & 100 \\
23 & - & - & - & - & - & - & - & -
\end{tabular}

TABLE IX

Solutions to G3 Problem (Minimize Carbon) Generation ADDITIONS IN MW

\begin{tabular}{c|c|c|c|c|c|c|c|c|}
$\mathrm{C}$ & 100 & 200 & 300 & 400 & 500 & 1000 & 2000 & 3000 \\
\hline 1 & 100 & 100 & 100 & 300 & 400 & 100 & 100 & - \\
2 & - & - & 100 & - & - & 800 & 800 & 100 \\
7 & - & 100 & - & 100 & 100 & 100 & 100 & 1000 \\
13 & - & - & 100 & - & - & 100 & 1500 & - \\
15 & - & - & - & - & - & - & - & 100 \\
16 & - & - & - & - & - & - & 900 & 100 \\
18 & - & - & - & - & - & - & - & 100 \\
21 & - & - & - & - & - & - & - & 100 \\
22 & - & - & - & - & - & - & - & - \\
23 & - & - & - & - & - & - & - & -
\end{tabular}

TABLE $X$

Solutions to G4 Problem (Minimize Carbon) Generation ADDITIONS IN MW

\begin{tabular}{c|c|c|c|c|c|c|c|c|}
$\mathrm{C}$ & 100 & 200 & 300 & 400 & 500 & 1000 & 2000 & 3000 \\
\hline 1 & - & 100 & 100 & 100 & 300 & 400 & 600 & 100 \\
2 & 100 & 100 & 100 & 100 & 100 & 100 & 100 & 100 \\
7 & - & - & - & - & 100 & 100 & 300 & 700 \\
13 & - & - & - & - & - & - & 800 & 1700 \\
15 & - & - & 100 & 100 & - & 100 & 100 & 100 \\
16 & - & - & - & - & - & 100 & 100 & 100 \\
18 & - & - & - & - & - & - & - & - \\
21 & - & - & - & 100 & - & 100 & - & 100 \\
22 & - & - & - & - & - & 100 & - & 100 \\
23 & - & - & - & - & - & - & - & -
\end{tabular}

TABLE XI

Solutions to Multi-Scenario Problem (Minimize Carbon) GENERATION ADDITIONS IN MW

\begin{tabular}{c|c|c|c|c|c|c|c|c|}
$\mathrm{C}$ & 100 & 200 & 300 & 400 & 500 & 1000 & 2000 & 3000 \\
\hline 1 & - & 100 & 100 & 100 & 100 & 400 & 800 & 100 \\
2 & - & 100 & 100 & 100 & 100 & 500 & 100 & 300 \\
7 & 100 & - & - & 100 & - & 100 & 100 & 900 \\
13 & - & - & - & 100 & 100 & - & 700 & 1200 \\
15 & - & - & - & - & 100 & - & 100 & 100 \\
16 & - & - & 100 & - & 100 & - & 100 & 100 \\
18 & - & - & - & - & - & - & - & - \\
21 & - & - & - & - & - & - & 100 & 300 \\
22 & - & - & - & - & - & - & - & - \\
23 & - & - & - & - & - & - & - & -
\end{tabular}


TABLE XII

Solutions to Multi-Scenario Problem (Minimize Carbon) CIRCUIT ADDITIONS

\begin{tabular}{c|c|c|c|c|c|c|c|c|}
$\mathrm{C}$ & 100 & 200 & 300 & 400 & 500 & 1000 & 2000 & 3000 \\
\hline$(1,2)$ & 0 & 0 & 0 & 2 & 0 & 0 & 0 & 0 \\
$(1,5)$ & 0 & 0 & 1 & 0 & 0 & 1 & 2 & 1 \\
$(1,8)$ & 0 & 0 & 0 & 0 & 0 & 0 & 0 & 1 \\
$(2,6)$ & 1 & 1 & 0 & 0 & 1 & 2 & 1 & 2 \\
$(3,24)$ & 1 & 1 & 1 & 1 & 3 & 0 & 3 & 0 \\
$(4,9)$ & 0 & 0 & 0 & 0 & 0 & 0 & 0 & 1 \\
$(5,10)$ & 0 & 1 & 0 & 0 & 0 & 1 & 0 & 0 \\
$(6,7)$ & 0 & 0 & 1 & 2 & 0 & 0 & 0 & 1 \\
$(6,10)$ & 2 & 1 & 1 & 2 & 2 & 0 & 0 & 0 \\
$(7,8)$ & 3 & 3 & 3 & 3 & 3 & 3 & 3 & 3 \\
$(9,12)$ & 0 & 1 & 0 & 0 & 0 & 1 & 0 & 0 \\
$(10,11)$ & 0 & 1 & 0 & 2 & 0 & 0 & 0 & 0 \\
$(10,12)$ & 1 & 1 & 2 & 0 & 1 & 0 & 0 & 0 \\
$(11,13)$ & 0 & 0 & 0 & 1 & 0 & 0 & 0 & 0 \\
$(12,23)$ & 0 & 1 & 1 & 1 & 1 & 0 & 0 & 0 \\
$(13,14)$ & 0 & 0 & 0 & 3 & 1 & 0 & 1 & 0 \\
$(13,23)$ & 0 & 0 & 0 & 1 & 0 & 0 & 0 & 0 \\
$(14,16)$ & 1 & 1 & 1 & 2 & 1 & 1 & 0 & 1 \\
$(11,23)$ & 0 & 0 & 0 & 0 & 0 & 1 & 0 & 0 \\
$(15,16)$ & 0 & 0 & 2 & 1 & 0 & 0 & 0 & 0 \\
$(15,21)$ & 0 & 3 & 1 & 1 & 2 & 3 & 1 & 1 \\
$(15,24)$ & 1 & 1 & 1 & 1 & 1 & 0 & 1 & 0 \\
$(16,17)$ & 3 & 1 & 1 & 1 & 1 & 1 & 1 & 1 \\
$(16,19)$ & 1 & 1 & 1 & 1 & 1 & 1 & 1 & 1 \\
$(16,23)$ & 1 & 0 & 1 & 0 & 0 & 0 & 1 & 0 \\
$(17,18)$ & 1 & 0 & 0 & 1 & 1 & 0 & 0 & 0 \\
$(20,23)$ & 0 & 1 & 0 & 0 & 0 & 0 & 0 & 0
\end{tabular}

